\title{
PREVALENCE, PATTERN AND OUTCOME OF PEDIATRIC LASSA FEVER DISEASE (LFD) IN A TERTIARY HOSPITAL, SOUTHEAST NIGERIA
}

Orji ML, Onyire NB, Unigwe US, Ajayi NA, Nwidi DU, Odey EC, Agboeze J, Ojide CK, Ujunwa FA, Onwe OE, Onoh RC, Madume P

\begin{abstract}
The prevalence and case fatality rates of Pediatric Lassa fever disease (LFD) are not well documented. This study was aimed at determining the prevalence, pattern and outcome of Pediatric LFD. It was a prospective observational study. A total of 183 subjects that met the criteria for LFD suspects were recruited consecutively and subjected to Lassa virus PCR test. Structured questionnaire was used to collect information. Of the 183 children recruited, 24 tested positive to Lassa virus PCR, giving a positivity rate of $13.1 \%$. Mean duration of illness at presentation was $8.54 \pm 3.83$ days. Fever, abdominal pain and vomiting were the three highest presenting complaints. Seven out of 24 children died giving a case fatality rate (CFR) of $29.2 \%$. Subjects with bleeding, poor urine output, convulsions and unconsciousness were more likely to die of LFD. Positivity and CFR of LFD are high. Improved case finding and prompt treatment is advocated
\end{abstract}

Key Words: Case fatality, Lassa virus, Pattern, Positivity rate, Outcome, Ribavirin 


\section{TEXT}

Lassa fever is an acute and sometimes severe viral hemorrhagic fever caused by Lassa virus; a single stranded RNA virus of the Arenaviridae family (1). Lassa fever is one of the viral hemorrhagic fevers that are rodent-borne. Mastomys natalensis is the species of rat responsible for the transmission of Lassa fever (2). Lassa fever is mostly seen in West African sub-region, where it causes over 5,000 deaths and 10-16\% admissions yearly, the observed case fatality among hospitalized patient is $15-50 \%$. $(3,4)$ In Nigeria, 17 states are said to be endemic for Lassa fever with Edo, Ondo and Ebonyi states having more than $75 \%$ of the cases reported and a case fatality rates of $14.6 \%, 24.2 \%$ and $23.4 \%$ respectively $(4,5)$.

Lassa virus is transmitted to humans through contact with contaminated food or household items that are contaminated by feces or urine of rodents. Transmission between humans can occur in either community or hospital settings, when there is contact of body fluid of an infected person. Nosocomial infections are common where there are inadequate hospital infection control measures (6).

The seroprevalence of Lassa fever in Nigeria is $21 \%$ (7). Akhuemokhan et al (8) studied prevalence of Lassa fever disease (LFD) in febrile children aged one month and above to 15 years of age in Irrua specialist hospital and reported a total prevalence rate of $5.7 \%$. Webb et al (9) carried out a similar study among children and reported a prevalence rate of $21 \%$. Of the 20 cases reported by Ajayi et al (10) in Ebonyi in a study carried out 9 years ago, only 2 (10\%) were between ages $10-19$ years.

Lassa fever has an incubation period of 3-21 days (1). Majority of human infections (about 80\%) with Lassa fever are mild or asymptomatic disease with symptomatic disease manifesting with 
non-specific symptoms. Lassa fever disease can masquerade as malaria, typhoid and pharyngotonsillitis $(10,11)$. McCarthy (12) observed four stages of disease. Starting with fever $\left(>39^{0} \mathrm{C}\right)$, general weakness, malaise in the first three days of illness then finally with coma and death after 14 days of illness. Akhuemokhan et al (8) reported vomiting and bleeding as symptoms that were significantly associated with an increased prevalence of LFD. Common symptoms of LFD as observed by Ajayi et al(10) in their study were fever (100\%), sore throat (70\%), abdominal pain (85\%), and vomiting (50\%), headache (35\%), body pain and weakness (25\%). Akpede et al (13) in their five case series of Lassa fever in children noted that the clinical features were myriad but fever was a constant feature while vomiting, gastritis and tonsillitis were common. Similarly Webb et al (9) reported that $60 \%$ of the children with LFD presented with fever, vomiting and cough. Features of shock, seizures, deafness and disorientation are seen in terminal illness and usually complications of the disease.

There is paucity of data on pediatric Lassa fever disease in endemic states and none from Ebonyi state. This study is therefore aimed at determining the epidemiology, clinical course and outcome of pediatric Lassa fever in Ebonyi state; it hopes to guide in better surveillance and management of children with Lassa fever 


\section{STUDY AREA}

Ebonyi State is located in the rain forest zone; the climate is tropical. The annual rainfall varies from $2,000 \mathrm{~mm}$ in the Southern areas to $1,150 \mathrm{~mm}$ in the north (14). The temperature throughout the year ranges between $21^{\circ} \mathrm{C}$ to $30^{\circ} \mathrm{C}$. It has two seasons, dry and wet. The dry season lasts from November to March while the rainy season lasts from April to October (14). It has a total population of 2,173,501 people, majority of which are Ibos (15).

Study design

The study is a hospital based observational study carried out in the children emergency room and the virology center of the Alex-Ekwueme Federal University Teaching Hospital Abakaliki (AEFUTHA), Ebonyi State, from January 2019 to January 2020.

\section{SAMPLE SELECTION}

All children aged 0-17 years admitted to newborn and Children emergency room with symptoms of unremitting fever for more than 2 days despite administration of anti-malarial and/or antibiotics \pm sore throat, bleeding from orifices and body pain were tagged as LFD suspects according to guideline by National Center for Disease Control (16). These LFD suspects were recruited consecutively to the study after obtaining informed consent from caregivers for the period of study. Blood samples from these patients were subjected to Lassa virus reverse transcriptase Polymerase chain reaction (RT-PCR) tests. The PCR was used to detect viral antigens in the child. Those infected with LFD as identified with positive PCR test were transferred to virology unit and followed up until discharge or death. Structured questionnaire was used to collect information on bio-data, socio-demographics, symptoms and signs at presentation, management given while on admission in virology center and outcome of the case. 


\section{ETHICAL CONSIDERATIONS}

Approval for the study was sought and obtained from the Health Research and Ethical Committee of Alex-Ekwueme Federal University Teaching Hospital Abakaliki (AE-FUTHA).

\section{DATA ANALYSIS}

The data obtained was entered into a spread sheet using the Microsoft excel 2007 and the analysis was done using the Statistical Package for Social Sciences version 19.0. Quantitative variables were summarized using means and standard deviations. Frequency tables were constructed as appropriate. The significance of associations between variables was tested using Chi-square test for comparison of proportion. Epi Info version 7.0 was used for multivariate analysis of data. The level of statistical significance was achieved if $p<0.05$.

\section{RESULTS}

Of the 183 LFD suspects recruited during the study period, 24 tested positive to Lassa PCR, giving a prevalence rate of $13.1 \%$. Mean duration of illness at presentation was $8.54 \pm 3.83$ days, range of 2 to 14 days. Table 1 shows that $41.6 \%$ of the Lassa fever infected children were within 6-12 years age bracket. Majority were females with a male to female ratio of 1:1.7 and children from lower social class were mostly affected.

All the infected children had a history of fever, while half of them reported history of contact with probable or confirmed Lassa fever patients. Abdominal pain and vomiting were second to fever in frequency of presentation with $41.7 \%$. A quarter of the children presented with convulsions, cough and dyspnoea, bleeding and poor urine output as shown in Table 2 
The pie chart in Figure 1 represents the outcome of Lassa fever disease; a total of 7 children died during the study period, giving a case fatality rate of $29.2 \%$. Three out of these deaths received their results post-mortem as such did not receive intravenous ribavirin medication. For the remaining 4 deaths, 3 occurred within 7 days on admission while the last occurred on the $8^{\text {th }}$ day on admission. Five (71.4\%) out of the 7 deaths were females, $3(42.8 \%)$ were less than 6 years of age, $2(28.6 \%)$ deaths within the 6-12 years age bracket and $2(28.6 \%)$ of the deaths were teenagers. There were no significant relationships between age and gender with outcome of Lassa fever disease in children $(\mathrm{p}=0.387$ and 0.562 respectively)

Majority $(87.5 \%)$ of the children that had Lassa fever disease received intravenous ribavirin medication. A total of $12.5 \%$ of the children that tested positive to Lassa virus PCR and were unable to receive ribavirin medication died as depicted on the pie chart in Figure 2

All the children that had abdominal pain, sore throat and headache were discharged. A total of $66.7 \%$ of the children who had convulsions died compared to $83.3 \%$ that did not have convulsions and were discharged home. Two-thirds of the children that had diarrhea and $70.0 \%$ that presented with vomiting were discharged home. There were significant relationships between symptoms such as abdominal pain and convulsions with or without coma and outcome of the Lassa fever disease ( $\mathrm{p}=0.008$ and 0.02 respectively) as shown in the Table $3 \mathrm{~A}$

Half of children that had cough in the course of illness and two-thirds of those with facial puffiness were discharged home. Majority $(83.3 \%)$ of children that bled and had poor urine output in the course of the Lassa fever illness died while $91.7 \%$ of children with a positive history of contact with a Lassa fever confirmed or probable case were discharged home. All the children $(100.0 \%)$ who did not receive intravenous ribavirin medication died of the illness and $4(19.0 \%)$ 
of the 21 children who received the drug died of the illness. The outcomes of Lassa fever disease among cases were significantly related to the presence of bleeding, poor urine out-put, positive history of contact with probable or confirmed case of the disease and commencement of ribavirin $(\mathrm{p}=0.001,0.001,0.025$ and 0.004 respectively) as shown in Table 3B

We did a multivariate analysis of variables and outcome. We found that children who presented with convulsion and coma were 10 times more likely to die from Lassa fever disease compared to other symptoms. Bleeding and poor urine output as symptoms had very high odds for death among Lassa fever infected subjects. Of the 21 children who received ribavirin, 3 (60.0\%) died within the first week of commencement of ribavirin while one death occurred after a week on ribavirin having developed complication of deafness. Subjects that completed ribavirin for more than 7 days were more likely to be discharged as shown in Table 4 


\section{TABLES}

Table 1: Socio-demographics of children with Lassa fever infection

\begin{tabular}{lll}
\hline Socio-demographics & Frequency & $\%$ \\
\hline Age (In years) & & \\
$<6$ & 7 & 29.2 \\
$6-12$ & 10 & 41.6 \\
$>12$ & 7 & 29.2 \\
Gender & & \\
Male & 9 & 37.5 \\
Female & 15 & 62.5 \\
Social class & 17 & 8.4 \\
Upper & 2 & 20.8 \\
Middle & 5 & 70.8 \\
Lower & & \\
\hline
\end{tabular}


Table 2: Pattern of Lassa fever disease presentation

\begin{tabular}{|c|c|c|}
\hline \multirow[t]{2}{*}{ Symptoms/Signs } & \multicolumn{2}{|c|}{ Frequency of Symptoms (\%) } \\
\hline & Yes & No \\
\hline Fever & $24(100.0)$ & $0(0.0)$ \\
\hline Sore throat & $4(16.7)$ & $20(83.3)$ \\
\hline Abdominal pain & $10(41.7)$ & $14(58.3)$ \\
\hline Convulsion/coma & $6(25.0)$ & $18(75.0)$ \\
\hline Headache & $3(12.5)$ & $21(87.5)$ \\
\hline Vomiting & $10(41.7)$ & $14(58.3)$ \\
\hline Diarrhoea & $3(12.5)$ & $21(87.5)$ \\
\hline Cough and dyspnoea & $6(25.0)$ & $18(75.0)$ \\
\hline Facial puffiness & $3(12.5)$ & $21(87.5)$ \\
\hline Yellowness of the eye & $2(8.3)$ & $22(91.7)$ \\
\hline Deafness & $1(4.2)$ & $23(95.8)$ \\
\hline Bleeding & $6(25.0)$ & $18(75.0)$ \\
\hline Poor urine output & $6(25.0)$ & $18(75.0)$ \\
\hline History of contact & $12(50.0)$ & $12(50.0)$ \\
\hline
\end{tabular}


Table 3A: Relationship between Clinical presentations and Outcome

Outcome $\quad X^{2} \quad p$ value

Symptoms/Signs $\quad$ Discharged (\%) Died (\%)

Sore throat

Yes

$4(100.0)$

$0(0.0)$

1.97

0.160

No

$13(65.0)$

$7(35.0)$

Abdominal pain

Yes

$10(100.0)$

$0(0.0)$

No

$7(50.0)$

$7(50.0)$

7.06

0.008

Convulsion/coma

Yes

$4(66.7)$

No

$15(83.3)$

3 (16.7)

5.45

0.020

Headache

Yes

3 (100.0)

$0(0.0)$

No

14 (66.7)

7 (33.3)

1.41

0.235

Vomiting

Yes

$7(70.0)$

$3(30.0)$

No

$10(71.4)$

4 (28.6)

0.01

9.39

Diarrhoea

Yes

$2(66.7)$

1 (33.3)

No

15 (71.4)

6 (28.6)

0.03

0.865 
Table 3B: Relationship between Clinical presentations and Outcome Cont'd

Outcome $\quad X^{2} \quad$ value

Symptoms/Signs Discharged (\%) Died (\%)

Cough and dyspnoea

Yes

$3(50.0) \quad 3(50.0)$

No

$14(77.8)$

$4(22.2)$

1.68

0.195

Facial Puffiness

Yes

2 (66.7)

1 (33.3)

No

15 (71.4)

6 (28.6)

0.03

0.865

Bleeding

Yes

1 (16.7)

$5(83.3)$

11.36

0.001

No

16 (88.9)

2 (11.1)

Poor urine output

Yes

1 (16.7)

$5(83.3)$

No

$16(88.9)$

2 (11.1)

11.36

0.001

History of contact

Yes

11 (91.7)

$1(8.3)$

No

$6(50.0)$

$6(50.0)$

5.04

0.025

Commenced Ribavirin

Yes

$17(81.0) \quad 4(19.0)$

No

$0(0.0) \quad 3(100.0)$

8.33

0.004 
Table 4: Multivariate analysis of covariates and Outcome

\begin{tabular}{|c|c|c|c|c|c|}
\hline \multirow[t]{2}{*}{ Covariates } & \multicolumn{2}{|l|}{ Outcome } & \multirow[t]{2}{*}{ Odd ratio } & \multirow[t]{2}{*}{$95 \% \mathrm{CI}$} & \multirow[t]{2}{*}{$p$ value } \\
\hline & Died & Discharged & & & \\
\hline \multicolumn{6}{|c|}{ Convulsion/coma } \\
\hline Yes & $4(66.7)$ & $2(33.3)$ & 10.00 & $1.2-81.81$ & 0.02 \\
\hline No & $3(16.7)$ & $15(83.3)$ & & & \\
\hline \multicolumn{6}{|l|}{ Bleeding } \\
\hline Yes & $5(83.3)$ & $1(16.7)$ & 40.00 & $2.96-539.67$ & 0.001 \\
\hline No & $2(11.1)$ & $16(88.9)$ & & & \\
\hline \multicolumn{6}{|c|}{ Poor urine output } \\
\hline Yes & $5(83.3)$ & $1(16.7)$ & 40.00 & $2.96-539.67$ & 0.001 \\
\hline No & $2(11.1)$ & $16(88.9)$ & & & \\
\hline \multicolumn{6}{|c|}{ Duration on Ribavirin } \\
\hline$\leq 7$ days & $3(60.0)$ & $2(40.0)$ & 22.50 & $1.51-335.35$ & 0.014 \\
\hline$\geq 8$ days & $1(6.3)$ & $15(93.7)$ & & & \\
\hline
\end{tabular}




\section{FIGURES}

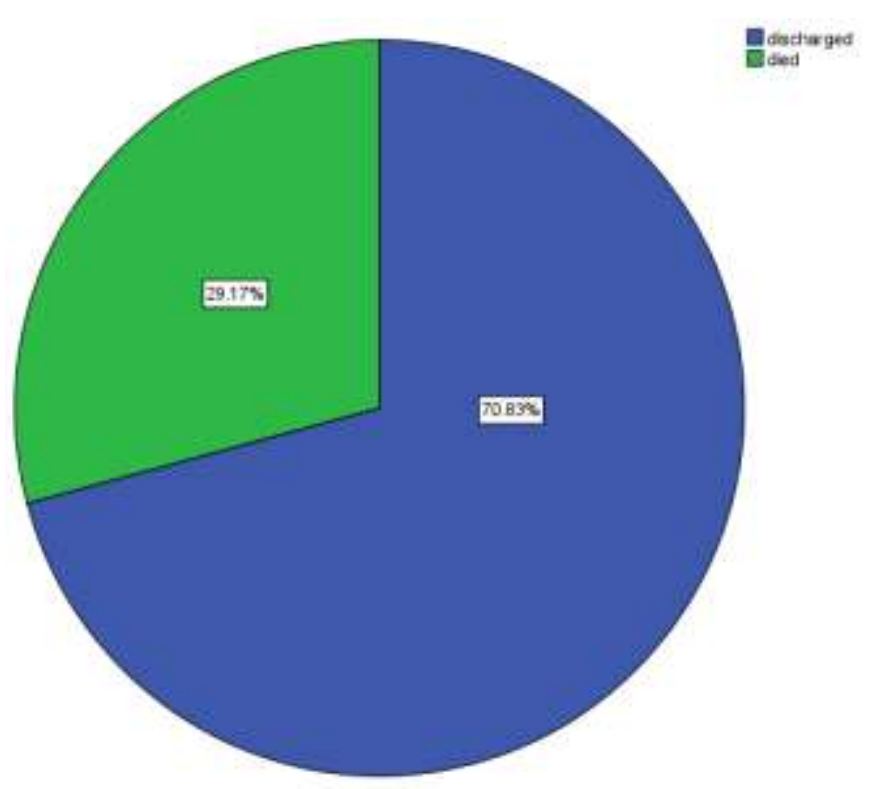

Figure 1: Pie chart showing the outcome of Children that had Lassa fever disease 


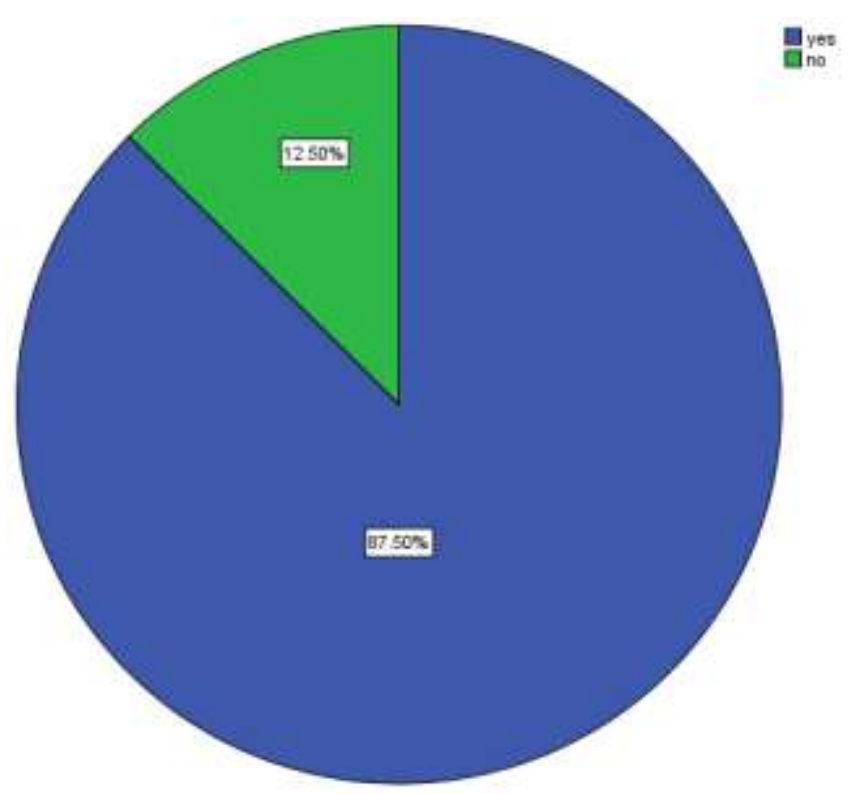

Figure 2 Pie chart showing the number of Lassa fever infected chiddren that received nibavirin medication 


\section{DISCUSSION}

Lassa fever disease positivity rate of $13.1 \%$ observed in children that met the case definition of Lassa fever suspect is rather high when compared to previously reported data by Ajayi et al (10) where only two paediatric cases were isolated. This may be explained by the study being a prospective study and focused on children unlike the study by Ajayi et al (10). Also increased availability of testing kit for Lassa fever disease and increasing awareness of disease symptoms by health care providers may be possible explanations to finding. More females than males were affected with LFD similar to that reported by Webb et al (9). It is possible that because females are more domesticated than males, there are more likely to have contact with the feces and urine of rodents or food and foodstuffs contaminated by urine and feces of rodents when engaging in house chores. Majority of the children infected by the Lassa virus were within the aged 6-12 age bracket. This is also similar to that reported by Webb et al (9).

Presenting symptoms were varied in index study similar to the experience of previous authors $(9$, 10, 13) However like most studies; a history of fever was a predominant finding in this study. This study noted abdominal pain and vomiting as common features after fever and studies by Ajayi et al (10) and Akpede et al (13) also reported sore throat, abdominal pain and vomiting as common presenting features following fever. Similarly Webb et al (9) reported that $60 \%$ of the children with LFD presented with fever, vomiting and cough. In all the above mentioned studies vomiting was a predominant finding followed by abdominal pain. This suggests that abdominal pain with vomiting in febrile children with unremitting fever spikes despite antimalaria and or antibiotic use should heighten the suspicion of the pediatrician working in Lassa fever endemic regions. Akhuemokhan et al (8) reported vomiting and bleeding as symptoms that were significantly associated with an increased prevalence of LFD. The abdominal pain found in index 
study were either localized or generalized in character, mimicking typhoid enteritis in most cases, appendicitis (two had surgery for appendicitis and was discovered to be LFD postoperatively) and hepatitis. The masquerading of LFD with non-specific symptoms may explain the average duration of illness of $8.54 \pm 3.83$ days before presentation at the facility. Dongo et al (17) in their case series noted that Lassa fever can present with features suggestive of acute abdomen underscoring the value of high index of suspicion when evaluating children at every entry point in the health facilities.

Of the 12 children that had a history of contact with a confirmed LFD patient, $91.7 \%$ of them were discharged home; only one died (8.3\%). While half of those without such history died on admission. The need for clinician to pursue and obtain history of previous contact confirmed or probable case of LFD patient if there is cannot be overemphasized. Such history when obtained early at presentation to a health facility can expedite the process of LFD case management, hence reducing morbidity and mortality for the patient and disease containment on the part of the healthcare provider, thereby reducing nosocomial spread to health workers.

A quarter of the children had bleeding from orifices and puncture sites, convulsions and eventually coma, poor urine output, cough and dyspnoea. These findings suggest that Lassa virus disease is multi-systemic in nature. Only one patient had deafness terminally before demise. This is similar to observation by Richmond and Baglole (18) Bleeding, poor urine output, convulsions and coma were ominous signs associated with poor prognosis. This was in tandem with that reported by Akpede et al (13) who noted that the presence of haemorrhage, acute renal failure, convulsion and coma were indictors of poor outcome. 
Of the 7 deaths recorded during the study period, three (3) died while waiting for the result of RT-PCR, diagnosis was made postmortem, hence did not receive ribavirin. Another three children died within four days of intravenous ribavirin medication while the last death occurred after 8 days of ribavirin from complications of deafness, seizures and coma. Although the average duration of illness before hospital presentation was long, it was observed that children who received ribavirin for more than 7 days had higher chances of recovery. Ribavirin remains the drug of choice in the treatment of LFD. It reduces death by $90 \%$ when given within the first six days of onset of symptoms (19). This was corroborated by previous authors that noted that patients on ribavirin were less likely to die compared to those who had no ribavirin $(6,10)$

The case fatality rate (CFR) observed in this study was $29.2 \%$. This may largely be attributed to delayed presentation as the mean duration of illness before presentation to the health facility was $8.54 \pm 3.83$ days. This is comparable to $15-50 \%$ CFR reported in endemic countries $(3,4)$. Prognosis for Lassa fever is generally good considering that $80 \%$ of infected persons have subclinical infection requiring no admission, however majority of mortalities occur among admitted patients with late presentation contributing largely to demise (14). Akhuemokhan et al (8) reported a case fatality rate of $23.1 \%$ in children with LFD while Ajayi et al (10) reported $30 \%$ case fatality among LFD subjects. Of the five cases reported by Akpede et al (13), mortality occurred in $40 \%$ of them while $60 \%$ fully recovered and were discharged.

More females compared to males died from complications of Lassa fever disease. This may be attributed to more females having the disease compared to males. Mortality was also observed more in children less than 6 years of age, although no significant relationships were found between age/sex and outcome of the disease. This is similar to finding by Yinka-Ogunleye et al (20) 
In conclusion, the positivity rate and case fatality rate of LFD in children observed in this study is rather high. Symptoms of fever, abdominal pain and vomiting with or without history of contact in children should heighten the index of suspicion for LFD by clinicians working in Lassa fever endemic area.

We wish to acknowledge the efforts of Medecins Sans Frontieres (Doctors without Borders) Organization in Lassa fever case management and infection prevention and control in AlexEkwueme Federal Teaching Hospital Abakaliki

Dr Maria-Lauretta Orji is a consultant Pediatrician working in the infectious Disease Unit of the Department of Pediatrics, Alex-Ekwueme Federal Teaching Hospital Abakaliki (AE-FUTHA). Her primary research interests are in infectious diseases. 


\section{REFERENCES}

1. Imported Lassa fever. New Jersey: Centers for Disease Control and Prevention (CDC) 2004. MMWR Morb Mortal Wkly Rep 2004;53(38): 894-7

2. Monath TP, Newhouse VF, Kemp GE, Setzer HW, Cacciapuoti A. Lassa virus isolation from Mastomys natalensis rodents during an epidemic in Sierra Leone. Science 1974; $185: 263-5$

3. World Health Organization. Lassa fever fact sheet [cited 2018 October 8] http://origin.who.int/mediacentre/factsheet/fs179.

4. Centers for Disease Control and Prevention. Lassa fever [cited 2018 April 23]. http://www.cdc.gov/vhf/lassa.

5. Ilori EA, Furuse Y, Ipadeola BO, Dan-Nwafor CC, Abubakar A, Womi-Eteng EO,et al. Epidemiologic and clinical Features of Lassa Fever Outbreak in Nigeria, January1 - May 6, 2018. Emerg Infect Dis 2019:25(6):1066-1074. Doi: 10.3201/eid2506.181035

6. Fischer-Hoch SP, Tomori O, Nasidi A, Perez-Oronoz GI, Fakile Y, Hutwagner L, et al. Review of cases of nosocomial Lassa fever in Nigeria: the high price of poor medical practice. BMJ 1995; 311:857-9

7. Tomori O, Fabiyi A, Sorungbe A, Smith A McCormick JB. Viral Hemorrhagic fever antibodies in Nigerian Populations. Am J Trop Med Hyg. 1988; 38: 407-10

8. Akhuemokhan OC, Ewah-Odiase RO, Akpede N, Ehimuan J, Adomeh DI, Odia I, et al. Prevalence of Lassa virus disease in Nigerian children with fever or without fever and convulsions in an endemic area. PLoS Negl Trop Dis 2017; 11(7): e0005711, doi:10.1371/journal.pntd.0005711 
9. Webb PA, McCormick JB, King IJ, Bosman I, Johnson KM, Elliot LH, et al. Lassa fever in Children in Sierra-Leone, West Africa. Trans R Soc Trop Med Hyg. 1986;80 (4): 57782

10. Ajayi NA, Nwigwe GC, Azuogu BN, Onyire NB, Nwonwu EU, Ogbonnaya LU, et al. Containing a Lassa fever epidemic in a resource-limited setting: Outbreak description and lessons learned from Abakaliki, Nigeria (January- March 2012). Int J Infect Dis 2013;17(11):e1011-6. Doi: 10.1016/j.ijid.2013.05.015

11. Nigeria Center for Disease Control. Standard operating procedures for Lassa fever case $\begin{array}{lllll}\text { management. } & \text { [Cited } & 2018 & \text { April }\end{array}$ http://www.ncdc.gov.ng/themes/common/docs/protocols/30_1502277315.pdf.

12. McCarthy M. USA moves quickly to push bio-defense research. Lancet 2002; 360:732

13. Akpede GO, Kayode-Adedeji BO, Dawodu SO. Manifestations and outcomes of Lassa fever in Nigerian children: a case series. Archives of Diseases in Children 2012; 97(1). [Cited 2018 June 18] https://adc.bmj.com/content/97/suppl_1/A38.3.

14. Weather/ climate of Ebonyi state. [Cited 2018 June 18] www.cometonigeria.com/search-by/ebonyi-state.

15. Federal Republic of Nigeria. 2006 Population and housing census of Nigeria. Federal Republic of Nigeria official gazette. Lagos 2007; 94. [Cited 2018 June 18] www.census.gov/geo/reference/urban.

16. Nigerian Centre for Disease Control. Standard operating procedures for Lassa fever case $\begin{array}{llll}\text { management } & \text { [Cited } & \text { October }\end{array}$ http://www.ncdc.gov.ng/themes/common/docs/protocols/30_1502277315.pdf. 
17. Dongo AE, Kesieme EB, Akpede GO, Okokhere PO. Lassa fever presenting as acute abdomen: a case series. Virol J 2013;10:123.

18. Richmond JK, Baglole DJ. Lassa fever: epidemiology, Clinical features and social consequences. BMJ. 2003; 327:1271-5

19. McCormick JB, King IJ, Webb PA, Scribner CL, Craven RB, Johnson KM, et al. Lassa fever effective therapy with ribavirin. N Engl J Med 1986; 314:20-6

20. Yinka-Ogunleye A, Ipadeola O, Saleh M, Ilori E, Dan-Nwafor C, Nwachukwu W, et al. 2016/2017 Lassa fever ouitbreak in Nigeria: age and sex have no effect on treatment outcome. Pan Afr Med J 2018; 8:3 